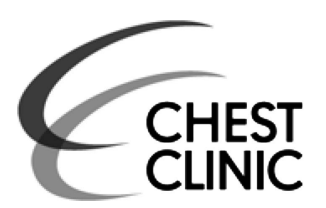

Correspondence to

Dr Martine Vrijheid, CREALCentre for Research in Environmental Epidemiology, Barcelona Biomedical Research Park (PRBB), Doctor Aiguader, 88; Barcelona 08003, Spain; mvrijheid@creal.cat

Received 10 April 2014 Revised 14 May 2014 Accepted 16 May 2014 Published Online First 6 June 2014

\title{
The exposome: a new paradigm to study the impact of environment on health
}

\author{
Martine Vrijheid
}

\section{ABSTRACT}

Environmental factors, here taken to include pollutants, lifestyle factors and behaviours, can play an important role in serious, chronic pathologies with large societal and economic costs, including respiratory disease. However, measurement of the environmental component in epidemiological studies has traditionally relied on much more uncertain and incomplete assessments than measurement of the genome. The 'exposome' has therefore been proposed as a new paradigm to encompass the totality of human environmental (meaning all non-genetic) exposures from conception onwards, complementing the genome. Evidently, there are large challenges in developing the exposome concept into a workable approach for epidemiological research.

These include: (1) the accurate and reliable measurement of many exposures in the external environment, (2) the measurement of a wide range of biological responses in the internal environment, and (3) addressing the dynamic, life course nature of the exposome. New tools and technologies that can be applied to address these challenges include exposure biomarker technologies, geographical mapping and remote sensing technologies, smartphone applications and personal exposure sensors, and high-throughput molecular 'omics' techniques. Prospective, population-based cohort studies have recently started to implement these methods using the exposome framework. The exposome thus offers a new and exciting paradigm for improvement and integration of currently scattered and uncertain data on the environmental component in disease aetiology. This should lead to a better understanding of the role of environmental risk factors in respiratory disease and other chronic pathologies, and ultimately to better primary prevention strategies.

Environmental factors, here taken to include environmental pollutants and lifestyle behaviours, are main causes of the chronic non-communicable diseases that dominate worldwide mortality, including cardiovascular disease, cancer and respiratory disease. The recent global burden of disease exercise has estimated that around $50 \%$ of all mortality worldwide was attributable to a few environmental factors, including indoor and outdoor air pollution, active and passive smoking and diet. ${ }^{1}$ At the same time, the aetiology of many common and highly complex chronic pathologies is still poorly understood. An example is asthma in which complex interactions between environmental, social and lifestyle factors (eg, ambient air quality, house dust, mould, smoking) and genetic and epigenetic factors are likely to underlie development and exacerbation. ${ }^{2}$
However, we have not been able to measure environmental factors with the same degree of accuracy and comprehensiveness as the genome. This is due to the many uncertainties in assessment of environmental exposures, which are traditionally measured through questionnaires and geographical mapping, and to the lack of studies that tackle multiple exposures; the environmental component in disease aetiology has thus far largely been studied using a 'one-exposure-onehealth-effect' approach.

\section{WHAT IS THE EXPOSOME?}

The 'exposome' was first proposed by Wild to encompass the totality of human environmental (meaning all non-genetic) exposures from conception onwards, complementing the genome. ${ }^{3}$ This concept was developed to draw attention to the need for better and more complete environmental exposure data, in order to balance the investment, tools and knowledge in genetics. Three overlapping domains within the exposome have been described as follows: (1) a general external environment to include factors such as the urban environment, climate factors, social capital, stress; (2) a specific external environment with specific contaminants, diet, physical activity, tobacco, infections, etc, and (3) an internal environment to include internal biological factors such as metabolic factors, gut microflora, inflammation, oxidative stress. ${ }^{4}$ There are large challenges in developing the concept of measuring every environmental exposure over a lifetime into a workable approach: a successful exposome would have to integrate many external and internal exposures from different sources continuously over the life course.

\section{THE EXTERNAL EXPOSOME}

In the exposome concept the challenge of accurate and reliable exposure assessment is multiplied, because it requires obtaining exposure data for many exposure variables. In the example of asthma this would mean accurate and simultaneous measurement of many factors related to the indoor environment (air pollutants, pets, mould, dust) and the outdoor environment (air pollution, green spaces, urban environment), as well as social and lifestyle factors. Within the external exposome, a distinction can be made between exposures in the specific external environment, including tobacco smoke, diet and chemical contaminants, which are traditionally largely assessed at the individual level by questionnaires, and exposures in the general external environment, such as outdoor air pollutants, noise and the urban environment, which are 
Figure 1 The exposome concept.

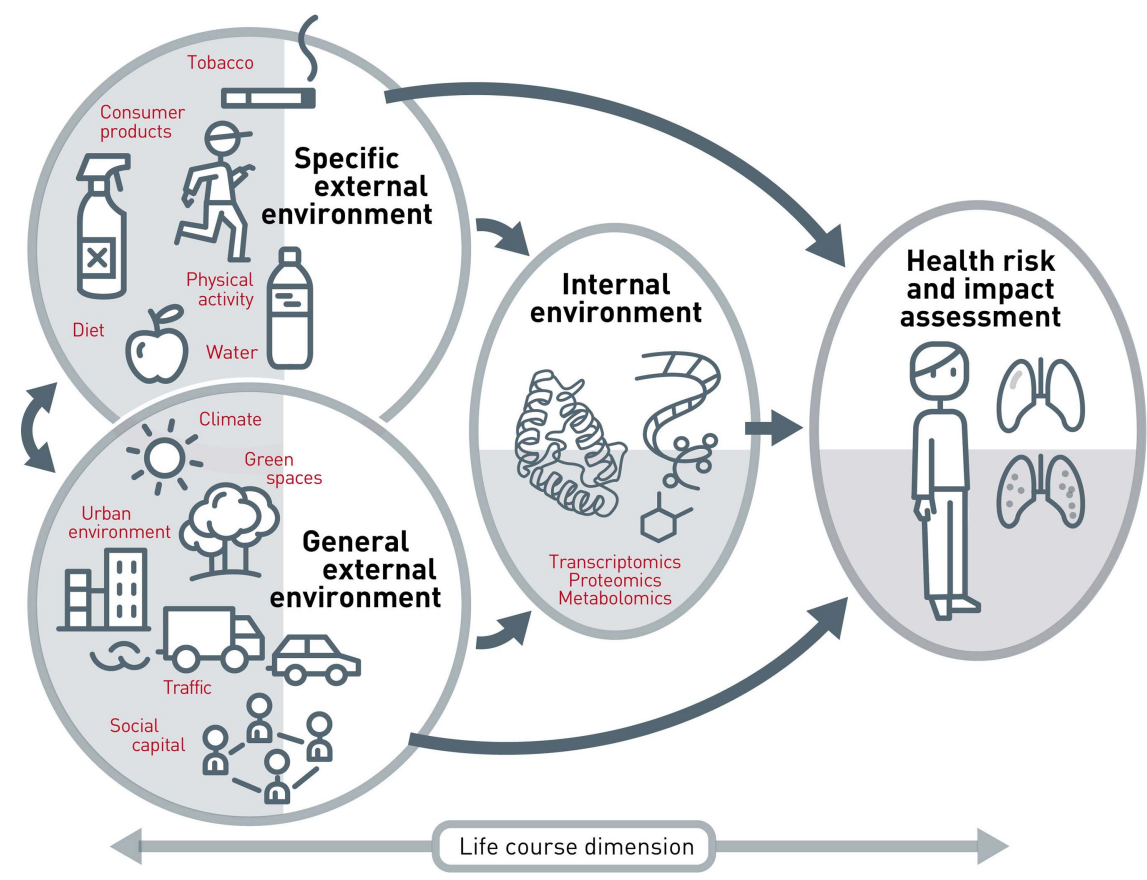

mainly assessed at the community level through geographical mapping methods (figure 1 ). ${ }^{5}$

For individual-assessed exposures, improvement in exposure assessment can be achieved through better modelling of exposure, for example by using predictive exposure models that combine questionnaire information with biomarkers and monitors for validation. ${ }^{5}$ For example, cotinine biomarkers may be used to validate questionnaire responses on environmental tobacco smoke, and indoor air pollution monitors may be used to validate questions on cooking and heating sources in the home. New smartphone-linked diaries and imaging are also promising tools for more accurate and complete exposure assessment, for example of diet and use of consumer products.

For community-level exposures, major improvements can be achieved by improving information on where people are, how they move through their environment, and, in case of air pollution, how much air they inhale. ${ }^{5}$ Knowledge about the inhalation rate, for example, may be integrated with personal air pollution measurements to estimate inhalation dose. For this, information about physical activity is needed and this can be collected through readily available accelerometers or sensors in smartphones. Further, people tend to move around in microenvironments: for example, near busy roads where exposures such as air pollutants or noise can be considerable higher compared with the rest of the time, or in parks where exposure to green space is higher and exposure to air pollution and noise is lower. Smartphone applications that integrate global positioning systems location data with physical activity information and pollution measurements, are now being developed to better characterise the external exposome. ${ }^{5}$

\section{THE INTERNAL EXPOSOME}

High-throughput molecular 'omics' techniques can analyse complete sets of biological molecules including smaller molecules (metabolomics), larger molecules (proteome) gene expression profiles (transcriptomics and epigenomics) and reactive electrophiles (adductomics). Recent years have seen the rapid development of omics applications in animal and in vitro experimental studies; for example, there are now numerous reports of epigenetic (methylation and miRNA) modifications arising from exposure to environmental toxicants. In human studies, applications have been extremely limited, but promising new results relate to their use to predict exposure-related health risks and individual susceptibilities, for example methylation profiles in relation to air pollution and cigarette smoking, metabolomic profiles in relation to diet, and protein profiling in relation to benzene, arsenic and lead.

In the development of the exposome concept, the contribution of omics techniques is likely to lie mainly in their potential to measure profiles (or signatures) of the biological response to complex exposure mixtures or a cumulative exposure experience. In particular, the hope is to find an unique matrix that could play an equivalent role to the DNA sequence in 'genomewide association studies', and allow the characterisation of the exposome without characterising each exposure separately. ${ }^{4}$ This is exactly the point where classical, single, exposure biomarkers have reached their limits: the measurement of numerous single analytes has limits in cost and available sample quantity. It is expected that the untargeted nature of omics data will capture biological responses to exposure in a more holistic way, provide clues about thus far unidentified risk factors, and give extra information on the molecular mechanisms underlying exposure-related health effects.

Further evaluation of omics tools for use in environmental health research requires careful attention to be paid to challenges relating to study design, validation, replication, temporal variance and metadata analysis. For example, for these techniques to be useful in larger studies, it has to be shown that intraindividual variability in the molecular profiles measured in systemic body fluids that can be collected in population studies (blood, urine) is less important than interindividual variability. Profiles of RNA transcripts, proteins and metabolites are highly variable over time; teasing out variations due to exposure changes remains a major challenge.

\section{THE DYNAMIC EXPOSOME}

The exposome is dynamic, as opposed to the static genome, and exposures vary on an hourly to yearly basis in the external and 
internal environments. Moreover, a given exposure or dose will not have the same effect during the various developmental periods that are critical to health and disease. Measurement of the exposome (internal or external) at one given point in time would not be sufficient to characterise all health impacts of the environment, but there are currently no prospective study populations that would allow lifelong repeated characterisation. One way forward would be to start developing the exposome at certain key points in exposure experience or disease development. ${ }^{4}$ For example, it is well recognised that the periods of organ development during prenatal life and infancy are especially vulnerable to the effects of environmental risk factors, which may manifest themselves throughout the lifetime in adult diseases including respiratory and allergic disease. Early-life may thus be a key point for defining the exposome and efforts are underway to accomplish this using prospective birth cohort studies. ${ }^{5}$ Another option is to apply the exposome concept to several cohorts that span the life course.

\section{ANALYSING THE EXPOSOME'S ASSOCIATION WITH HEALTH}

Collection of data on many chemical and physical exposures and on molecular omics profiles generates huge amounts of data which have to be stored, managed, analysed and interpreted. Statistical analyses of environmental risk factors have traditionally used regression models suitable for one-exposure-onehealth-effect type analyses. Approaches that may prove useful to evaluate multiple and combined exposures include: (1) the evaluation of risk estimates for many single exposures in an agnostic, hypotheses generating manner. This so-called 'environment-wide association study' approach has been used to discover priority risk factors for type 2 diabetes mellitus and all cause mortality ${ }^{6}$; $(2)$ the evaluation of risk estimates for combined exposures through data-driven dimension reduction methods; and (3) the evaluation of risk estimates for groups of subjects sharing a similar exposome, such as in a Bayesian profile regression analysis. Once risks related to the exposome have been estimated, systematic impact assessments of health effects are needed to inform the development of policies.
Health impact assessments aim at providing decision-makers with sound information on policy implications for health. Traditional policy development tends to be one dimensional, focusing on one or a limited number of risk factors and one or a limited number of health outcomes. Tools are now under development that try to capture the complexity of multiple exposures and health outcomes and integrate risk and benefits in a more holistic model.

\section{RELEVANCE FOR THE CLINICIAN}

The exposome concept offers a new and exciting paradigm for improvement and integration of currently scattered and uncertain data on the environmental component in disease aetiology. Complementing the 'one-exposure-one-health-effect' approach of current aetiological studies with a more holistic approach to the environmental component is essential to improve our understanding of the predictors, risk factors and protective factors of complex, multifactorial, chronic pathologies, including respiratory disease. Better understanding will ultimately lead to better prevention strategies.

Competing interests None.

Provenance and peer review Commissioned; internally peer reviewed.

\section{REFERENCES}

1 Lim SS, Vos T, Flaxman AD, et al. A comparative risk assessment of burden of disease and injury attributable to 67 risk factors and risk factor clusters in 21 regions, 1990-2010: a systematic analysis for the Global Burden of Disease Study 2010. Lancet 2012;380:2224-60.

2 Martinez FD. Genes, environments, development and asthma: a reappraisal. Eur Respir J 2007;29:179-84.

3 Wild CP. Complementing the genome with an "exposome": the outstanding challenge of environmental exposure measurement in molecular epidemiology. Cancer Epidemiol Biomarkers Prev 2005;14:1847-50.

4 Wild CP. The exposome: from concept to utility. Int J Epidemiol 2012:41:24-32.

5 Vrijheid M, Slama R, Robinson 0, et al. The Human Early-Life Exposome (HELIX): project rationale and design. Environ Health Perspect Published Online First: 7 Mar 2014. doi:10.1289/ehp.1307204

6 Patel CJ, Rehkopf DH, Leppert JT, et al. Systematic evaluation of environmental and behavioural factors associated with all-cause mortality in the United States National Health and Nutrition Examination Survey. Int J Epidemiol 2013;42:1795-810. 